Acta Physica Academiae Scientiarum Hungaricae, Tomus 24 (4), pp. 433-435 (1968)

\title{
ON LEVINSON'S THEOREM WHEN GHOSTS ARE PRESENT
}

By

\author{
K. L. NAGY \\ INSTITUTE FOR THEORETICAL PHYSICS, ROLAND EÖTVÖS UNIVERSITY, BUDAPEST
}

(Received 26. IX. 1967)

1. It is well known from Levinson's theorem [1] for potential scattering that the scattering phase difference between threshold and infinite energies

$$
\delta_{\text {thresh }}-\delta_{\infty}=\pi\left(N-N_{e}\right),
$$

where $N$ is the number of the eigenstates of the total, $N_{e}$ is that of the number of the unperturbed Hamiltonian. Thus, $N-N_{e}$ is the number of bound states. This result cannot be prooved for elementary particle physics, but it has been checked for several different models (for references see e.g. [2]). Moreover, general arguments based on the $N$ over $D$ method have also been given [3] explaining why this statement should also hold here. All the field theoretical models treated show the characteristic, however, that the number of interpolating fields is equal to the number of real particles. We wish to check the statement in a model in which this is not so; in the LEE model with ghosts.

2. In the LEE model with cut-off [4] (i.e. without ghosts) the $N-\Theta$ scattering phase shift difference for stable $V$ particle

$$
\delta\left(\boldsymbol{m}_{\theta}\right)-\delta(\infty)=\mathbf{0},
$$

showing that the (intermediate) $V$ particle can be considered as an elementary one. Turning to the case without cut-off and for consistency introducting indefinite metric at the same time one gets for the invariant amplitude [5]

$$
\begin{gathered}
\boldsymbol{t}(\omega)=\frac{\omega}{k} \boldsymbol{e}^{i \delta(\omega)} \sin \delta(\omega)=-\frac{\pi \omega}{2 \boldsymbol{h}^{+}(\omega)}, \\
k=\sqrt{\omega^{2}-\boldsymbol{m}_{\Theta}^{2}},
\end{gathered}
$$

where $h^{+}(\omega)$ is the value of the function

$$
\begin{aligned}
& h(z)=a+b z+G(z) z^{2}, \\
& G(z)=\int \frac{k^{2} d k}{2 \omega(\omega-z)},
\end{aligned}
$$


$a, b$, finite parameters, on the $+i$ side of the cut from $m_{\Theta}$ to $+\infty$. From this, either by noticing that from (2)

$$
\begin{gathered}
\operatorname{ctg} \delta(\xi)=\frac{A}{\sqrt{\xi^{2}-1}}+B \frac{\xi}{\sqrt{\xi^{2}-1}}+C \log \left(\xi+\sqrt{\xi^{2}-1}\right), \\
\\
A+B<0, \quad C>0, \xi=\frac{\omega}{m_{\theta}} .
\end{gathered}
$$

or by using the following

Theorem: let $D(z)$ be a meromorphic function on the complex $z$ plane cut along the real axis from $\mu$ to $+\infty$. Then the value of the integral

$$
I=\frac{1}{2 \pi i} \int_{C} \frac{D^{\prime}(z)}{D(z)} d z
$$

for the closed curve shown in the Figure is given by

$$
I=\sum_{\text {zeros }} M_{i}-\sum_{\text {poles }} N_{j}
$$

where $M_{i}$ is the order of the $i$-th zero, $N_{j}$ is the order of the $j$-th pole of $D(z)$ lying inside $C$

one gets for all the special cases of discrete, complex (two first order zero), or dipole ghost (one second order zero),

Here

$$
\delta\left(m_{\Theta}\right)-\delta(+\infty)=\pi
$$

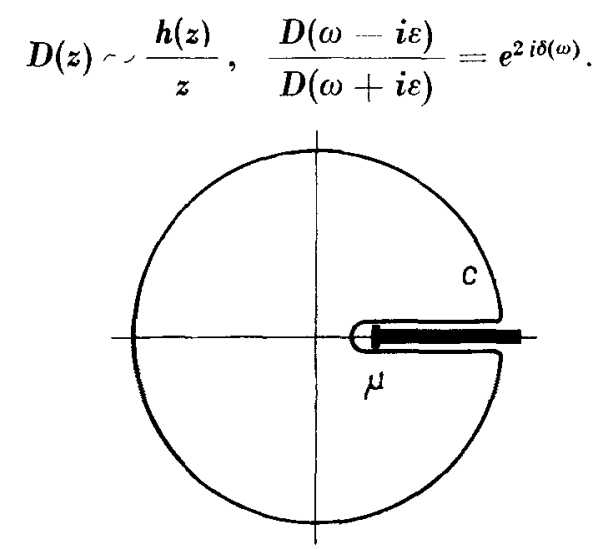

It is worth while mentioning that in $D(z)$ a CDD pole corresponds to $N_{e}(=1)$ in (1), the appearance of which is usually associated with an elementary particle. Furthermore (3) indicates a bound state. Thus, one is inclined to 
say that the ghost is of dynamic origin. As to which state is dynamic in reality, however, cannot be answered. This is quite clear considering e.g. the case of the complex ghosts. They certainly are treated on the same footing from every point of view. Moreover (3) indicates also the dipole ghost, which is not an eigenstate of the total Hamiltonian. This shows that in the case of indefinite metric the phase shift difference is associated not with the eigenstates but rather with the principal linear manifolds of the Hamiltonians.

One can get the result (3) for the modified model [6] where an extra field also has been introduced. There, one finds two CDD poles corresponding to the elementary fields and three states.

The values of the renormalization constants $Z_{i}$ defined by

$$
Z_{i}^{1 / 2}=\left\langle 0\left|\varphi_{v}\right| E_{i}\right\rangle
$$

where $\left|E_{i}\right\rangle$ are any of the states normal ghost, or dipole ghost, $\left|Z_{i}\right|$-s are logarithmically divergent. (We have no $0 \leqslant Z \leqslant 1$ ). Presumably, this is a consequence of the supposition that the mass equation to a single field should have two roots, which in turn implies indefinite metric [7]. Anyway, ghost or no ghost, (3) indicated an extra state here as expected.

\section{REFERENCES}

1. N. Levinson, Kgl. Danske Videnskab. Selskab, Mat. Fys. Medd., 25, No. 9, 1949.

2. B. Div, Qu'est-ce qu'une particule élémentaire? Masson Cie, Paris, 1965.

3. P. E. Kaus and F. Zachaniasen, Phys. Rev., 138 B, $1304,1965$.

F. Zachartasen, Leeture Notes on Bootstrap, Pacific International Summer School, 1965.

4. M. T. Vaughe, R. Aron and R. D. Amado, Phys. Rev., 124, 1258, 1961.

5. W. Hersenberg, Nuclear Physics, 4, 532, 1957.

6. K. L. NAGY, Nuovo Cimento, 17, 384, 1960.

7. H. Umezawa, Quantum Field Theory, North-Holland P. C. Amsterdam, 1956. 